\title{
Taking the heat out of organ donation
}

Authors: Ina Jochmans ${ }^{1}$ and Christopher J. E. Watson ${ }^{2}$

\section{Affiliations:}

1. Abdominal Organ Transplantation University Hospitals Leuven and Department of Microbiology and Immunology, KU Leuven, Leuven, Belgium.

2. University of Cambridge Department of Surgery and NIHR Cambridge Biomedical Research Centre, Cambridge, UK.

\section{Word count: 749}

The results of transplantation vary depending on the quality of the organ and the nature of the donor. In 2012 in the US, 73\% of kidneys from deceased organ donors were functioning 5 years later, compared to $84 \%$ of kidneys from living donors. ${ }^{1}$ One of the explanations for the superior results of living donor kidney transplantation is the avoidance of the catastrophic physiological changes that accompany brain death in the deceased organ donor.

Brain death has complex and widespread consequences that affect all organs. The reflex autonomic response to increasing intracranial pressure includes release of massive quantities of catecholamines causing peripheral vasospasm and reduced organ perfusion. As brain death evolves, haemodynamic instability worsens with loss of sympathetic vascular tone and hypovolaemia as a consequence of diabetes insipidus.

Brain death also results in a range of inflammatory and immune responses. The complement system is activated and pro-inflammatory cytokines such as interleukins-1 and -6, tumour necrosis factor and gamma interferon are released. ${ }^{2}$ Endothelial activation results in upregulation of human leucocyte antigens, selectins, and other adhesion molecules. The coagulation system is also activated by 
thromboplastins released from the brain, and in rat models fibrin deposition has been observed in peritubular and glomerular capillaries. ${ }^{3}$

It is against this background of haemodynamic and pro-inflammatory events that organ donation takes place. In kidney transplantation, the acute kidney injury that follows donor death, organ preservation, and graft implantation commonly manifests as a delay in achieving independence from dialysis, termed delayed graft function. ${ }^{4}$ The incidence of delayed graft function is greatest (up to 50\%) in recipients of older, extended criteria kidneys. Not only is delayed graft function costly in terms of increased inpatient stay and the need for dialysis, but it is also associated with a higher risk of acute graft rejection and early graft loss..$^{4,5}$

In this issue of the Journal Niemann and colleagues report a simple intervention in the brain-dead donor that significantly reduced delayed kidney graft function (ClinicalTrials.gov number, NCT01680744). ${ }^{5}$ Organ donors were randomised either to be maintained at normothermia (36.5$37.5^{\circ} \mathrm{C}$, the current clinical standard) or to undergo modest cooling $\left(34-35^{\circ} \mathrm{C}\right)$. Cooling was reached within 4 hours of enrolment, and its median duration was 16.9 hours until organs were recovered from the donor. The effect of this intervention was a significant reduction of the need for dialysis within the first 7 days post-transplant from $39.2 \%$ in kidneys from normothermic control donors to $28.2 \%$ in kidneys from hypothermic donors (adjusted odds ratio $0.62,95 \%$ confidence interval $0.43-0.92$ ). The beneficial effect was greatest in the subset of extended criteria donor kidneys ( $31.0 \%$ versus $56.5 \%$ in kidneys from hypothermic and normothermic donors, respectively). The trial was stopped following an interim analysis showing clear efficacy, at which point 370 donors had been enrolled with 583 kidneys transplanted.

Therapeutic hypothermia, such as that employed in the study of Niemann et al., ${ }^{5}$ is commonly used to limit neurological damage in patients who have suffered ischaemic or traumatic brain injury. Hypothermia reduces metabolic rate and blood flow to the brain, reduces oxygen consumption and glucose metabolism, and preserves high-energy phosphates such as ATP. ${ }^{6,7}$ In addition it reduces 
apoptosis, free radical production, and release of pro-inflammatory cytokines, as well as impairing neutrophil and macrophage function. Hypothermia may thus decrease the primed inflammatory state that occurs in kidneys from brain-dead donors. ${ }^{8}$ Cooling also affects coagulation, favouring thrombolysis and reducing fibrin formation, factors which may reduce the occurrence of microthombi that have been implicated in delayed graft function. ${ }^{9}$

The Niemann study does have some shortcomings. First, the observed effects relate to initial graft function, with no information on acute rejection or the potential longer term effects on graft survival. Neither does the study report on the outcomes of other organs, such as livers and pancreases, from these donors. Lastly, the kidneys from hypothermic donors were transplanted with a shorter cold ischaemic time, which may have contributed to the observed effect. Nevertheless the authors are to be congratulated for introducing such an intervention in the organ donor. Such trials are difficult to perform because of complex ethical, regulatory and logistical concerns. ${ }^{10,11}$

The results reported here will be welcomed for many reasons, not least because they have shown that, in this era of high technology medicine and targeted drug therapy, it is still possible to identify a simple, cheap intervention that can have dramatic therapeutic effects. Further investigation is required to determine how long donors need to be cooled before donation, how soon after brain death the intervention is effective, and the mechanisms underlying the effect. 


\section{References}

1. Matas AJ, Smith JM, Skeans MA, et al. OPTN/SRTR 2012 Annual Data Report: kidney. Am J Transplant 2014;14 Suppl 1:11-44.

2. Floerchinger B, Oberhuber R, Tullius SG. Effects of brain death on organ quality and transplant outcome. Transplantation Rev 2012;26:54-9.

3. Morariu AM, Schuurs TA, Leuvenink HG, van Oeveren W, Rakhorst G, Ploeg RJ. Early events in kidney donation: progression of endothelial activation, oxidative stress and tubular injury after brain death. Am J Transplant 2008;8:933-41.

4. Siedlecki A, Irish W, Brennan DC. Delayed graft function in the kidney transplant. Am J Transplant 2011;11:2279-96.

5. Niemann CU, Feiner J, Swain S, et al. Therapeutic Hypothermia in Deceased Organ Donors on Delayed Graft Function. N Engl J Med 2015; accepted.

6. Polderman $\mathrm{KH}$. Mechanisms of action, physiological effects, and complications of hypothermia. Crit Care Med 2009;37:S186-202.

7. Yenari MA, Han HS. Neuroprotective mechanisms of hypothermia in brain ischaemia. Nat Rev Neurosci 2012;13:267-78.

8. de Vries DK, Lindeman JH, Ringers J, Reinders ME, Rabelink TJ, Schaapherder AF. Donor brain death predisposes human kidney grafts to a proinflammatory reaction after transplantation. Am J Transplant 2011;11:1064-70.

9. Turunen AJ, Lindgren L, Salmela KT, Kyllonen LE, Petaja J, Pesonen EJ. Intragraft coagulation events and delayed graft function in clinical renal transplantation. Transplantation 2008;85:693-9.

10. Feng S. Donor intervention and organ preservation: where is the science and what are the obstacles? Am J Transplant 2010;10:1155-62.

11. Warrens AN, Lovell H, Committee UKDE. Addressing problems in transplantation research in the United kingdom. Transplantation 2012;93:860-4. 